\title{
Compendium of vascular variations in the anterior cervical region and right up- per limb - A Case Report
}

${ }^{1}$ Udhayakumar S, ${ }^{2}$ Kalaiyukan S, ${ }^{2}$ Srishankar

${ }^{1}$ Faculty of Medicine, Jaffna, ${ }^{2}$ Teaching Hospital, Jaffna

\begin{abstract}
It is axiomatic that knowledge of variations of the major vessels is crucial, not only from anatomical standpoint, but also clinically. During the routine dissection for undergraduate study in Department of Anatomy, Faculty of Medicine, University of Jaffna, Sri Lanka, we came across compendium of vascular variations. Brachiocephalic trunk originated to the left of midline and its branches, the right common carotid and subclavian arteries, crossed the cervical trachea in front from left to right just above the left brachiocephalic vein. The right axillary artery divided into radial and ulnar arteries at the level of lower border of the teres major and these two branches travelled between the biceps brachial and median nerve. Radial artery took the course of the brachial artery in the arm and cubital fossa. Ulnar artery passed in a deeper plane in the arm medial to the biceps brachii and radial artery and passed deep to pronator teres and continued in the forearm lateral to the ulnar nerve as usual. The lateral root of median nerve crossed in front of axillary to unit with the medial root to form the median nerve, which coursed medial to the radial and ulnar arteries in the brachium The aim of this case reportis to compare with the variations reported and to discuss its clinical implications. In conclusion, we present a report of multiple arterial variations in the anterior cervical region and upper limb. Correct identification of variations is necessary for clinical practice, appropriate invasive techniques and to avoid complications.
\end{abstract}

\section{Key Words}

brachiocephalic trunk, right common carotid artery, right subclavian artery, radial artery, ulnar artery

\section{Introduction}

The brachiocephalic trunk is the first branch of the arch of aorta usually arises from it in or a little to the left of the midline of the body within the superior mediastinum. It slopes upwards across the trachea to the back of the right sternoclavicular joint, where it divides into the right common carotid and right subclavian arteries. The termination of the brachiocephalic vein lies in front of the brachiocephalic trunk. Brachial artery is a major artery of brachium and it is formed at the inferior border of teres major as the continuation of axillary artery. It ends at the level of the neck of the radius by dividing into its two terminal branches, ulnar and radial arteries. At the level of the mid arm, the median nerve crosses anterior to brachial artery from lateral to medial side (1)

\section{Case Report}

During the routine dissection for undergraduate study in Department of Anatomy, Faculty of Medicine, University of Jaffna, Sri lanka, we came across variation in the arterial supply of upper limb of a 60 -year-old female cadaver. The brachiocephalic trunk originated from the arch of aorta to the left of trachea and ascended a short course to the left of the trachea and divided into right common carotid artery and subclavian artery to the left of trachea. These two branches made a $90^{\circ}$ angle with the parent vessel and traversed in front of cervical trachea in the neck from left to right above the left brachiocephalic vein (Figure 1).

The right common carotid artery crossed at the level of $7^{\text {th }}$ trachea ring and the right subclavian artery just below it. The right common carotid artery passed upwards to the right of trachea (Figure 1). Right subclavian artery passed over the $1^{\text {st }}$ rib and continued as axillary artery in the axilla. At the lower border of teres major, it divided into radial and ulnar arteries. The radial artery had superficial course in the arm along the medial aspect of biceps brachii and passed deep to the bicipital aponeurosis, 
similar to the course of brachial artery (Figure 2). The remaining course of the radial artery in the forearm and hand was as usual. Ulnar artery passed in a deeper plane in the arm medial to the biceps brachii and radial artery. In the cubital fossa, ulnar artery passed deep to pronator teres and continued in the forearm lateral to the ulnar nerve as usual (Figure 3). The lateral root of median nerve crossed in front of the $2{ }^{\text {nd }}$ part of axillary and united with the medial root and the median nerve is formed medial to the axillary artery (Figure 2). The median nerve coursed medial to the radial and ulnar arteries in the brachium (Figure 2).
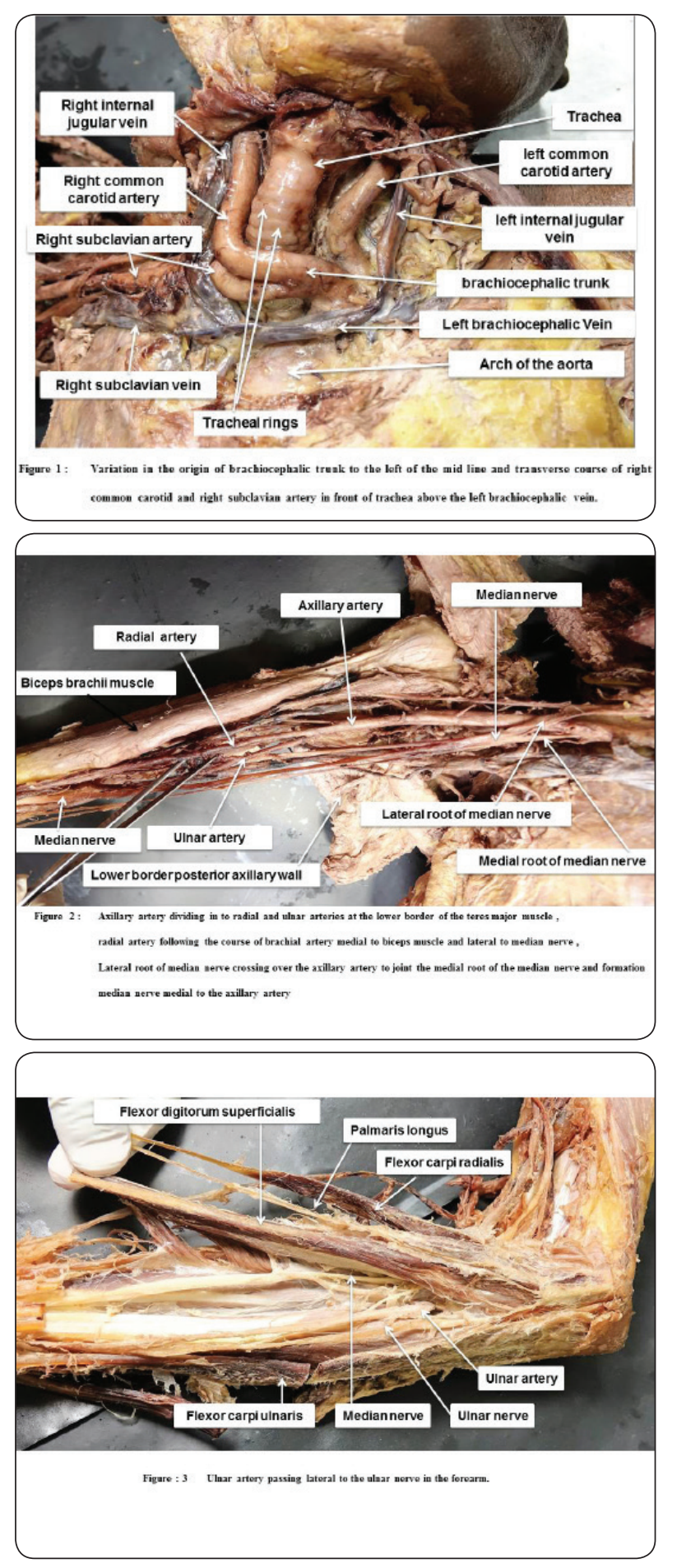

Vol.32, No.1, July 2020

\section{Discussion}

Variation in the origin, course and terminal branching pattern of brachiocephalic trunk in relation to the trachea, thyroid and other structures has a profound clinical impact.

This Case describes the origin of brachiocephalic trunk to the left of midline and a very short course to the left of trachea before diving into its terminal branches. Even though this has been reported by some authors, it is a rare variant. In a study by Budhiraja et al (2) on anatomical variations in the branching pattern of human arch of aorta on 52 cadavers, 2 cases $(3.8 \%)$ showed point of origin of the brachiocephalic trunk to the left of midline. Here the brachiocephalic trunk crossed obliquely upwards in front of trachea from left to right. Gaikwad et al (3) also reported a similar case, in which the brachiocephalic trunk crossed in front of trachea transversely from left to right along the lower pole of thyroid gland in the neck region. They also added that the respiratory compromise due to this variant brachiocephalic trunk / innominate artery (IA) is referred as IA compression syndrome.

Iterezote et al (4) performed neck dissection in 110 formalin fixed specimens to study the variations in the arch of aorta. They found variation in only one cadaver $(0.9 \%)$, where abnormally wide (19 $\mathrm{mm}$ ) brachiocephalic trunk bifurcated anterior to the trachea into its terminal branches. Here right common carotid artery ascended very closely along the right lobe of thyroid gland.

Crossing of the terminal branches of the brachiocephalic trunk across the trachea as in our case is a very rare variant. Sharma \& Gurud (5) describes the anomalous course of right common carotid and subclavian arteries which is similar to our report. In addition to pre-tracheal (subisthmic) course of right subclavian and common carotid arteries they found a high arch of aorta, the summit of which lay level with the jugular notch and an abnormally wide brachiocephalic trunk overlapping left half of cervical trachea. Karunakaran et al (6) reported an anomaly during surgery of multinodular goiter, they found right common carotid artery abnormally superficial and crossing the lower pole of thyroid gland because the brachiocephalic trunk arises from the arch of aorta near the left sternoclavicular joint, they added that the surgery would have ended in vascular 
catastrophe, if they have not been meticulous.

A 90 degree turn of major vessels (common carotid artery and subclavian artery) as in the present case lead to deprivation of the blood supply to brain and important structures of head and neck.

Any variation in the great arteries may produce respiratory distress in paediatric patients and two major arteries crossing trachea may cause tracheomalacia (5). Thorough knowledge of the aortic branches is important in operative procedures of anterior neck structures like tracheostomies, thyroidectomies and mediastinoscopies is needed. Distortion of the normal vascular anatomy increases the risk of injury to these vessels unless the clinician is aware of such possibilities and exercise great caution especially in percutaneous procedures (5). Careful inspection and palpation of suprasternal region to exclude any pulsatile mass prior to any intervention will decrease the risk of accidental damage to aberrant vascular anatomy. At present diagnostic ultrasound is used to detect the presence of anatomic variations of the vessels before percutaneous procedures avoiding inadvertent injuries (4). Therefore, scanning of anterior cervical region prior to any surgical intervention should be encouraged.

Present report describes a division of axillary artery at the lower border of teres major into radial and ulnar arteries with the absence of brachial artery. Radial artery had a superficial course in the arm deep to the deep fascia, similar to the course of brachial artery and followed the normal course in the forearm and hand. Variations in the origin and course of brachial, radial and ulnar arteries are not uncommon and have been described by many researchers.

High origin of radial and ulnar artery forms the highest percentage of variations of brachial artery. High origin of radial artery occurrence is 3 to 15 $\%(7 \& 8)$ and that of ulnar artery is $1 \%(7)$. High division of brachial artery into its terminal branches may occur at any point in the normal course of the vessel, but it is more common in the middle third (7). If the two vessels run parallel to each other to the elbow in the usual position of the brachial artery and from this point, one branch follows the normal course of radial artery and the other one takes the normal course of the ulnar artery, this arrangement is considered a simple high division of the brachial artery (7).

Teli et al (7) reported a case where the brachial artery divided into its terminal branches in the upper third of the arm, about $1.5 \mathrm{~cm}$ distal to the lower border of teres major. They classified the variation as superficial brachioradial artery, which is high origin of radial artery with course superficial to forearm flexors. Calisto et al (9) also reported a case of high division of axillary artery into radial and ulnar arteries, where the radial artery followed a superficial path in the arm, remaining outside the brachial conduit, superficial to the deep fascia and along the lateral edge of biceps brachii in the arm. In their study ulnar artery followed the path of the absent radial artery. High division of brachial artery in the upper third of the arm has been reported by Jadhav \& Pawar (8). In their study both the radial and ulnar arteries had superficial course medial to the biceps brachii and median nerve crossed the radial artery anteriorly from lateral to medial side in the mid-arm. After that the median nerve coursed between radial and ulnar arteries. They stated bifurcation of brachial artery above the level of the line joining the two epicondyles is termed as high division (8).

Even though variations in the upper limb arterial pattern have been documented by several authors, awareness on those variations has major clinical impact. Variations in blood vessels' branching pattern, position or course can alter routine clinical procedures. Brachial artery is clinically important because it is used for recording blood pressure $(8$ \& 9). pulsed doppler sonographic measurements and arteriography of different parts of the body (8). They also can create difficulties in complex interventions, such as cardiac catheterization, flap surgery or amputations (9). Variation in the arteries of the arm and their superficial position make the arteries more vulnerable to trauma and bleeding. On the other hand more accessible for arteriovenous fistula creation (9)

In conclusion, we present a cadaveric case of multiple arterial variations in the anterior cervical region and upper limb. Correct identification of these variations is necessary for clinical practice, appropriate invasive techniques and to avoid complications. 


\section{References}

1. Author. Sinnatamby, C S. Last's anatomy: regional and applied, 12th ed. Elsevier, 2013:51-54, 191-193.

2. Budhiraja V, Rastogi R, Jain V, Bankwar V, Raghuwanshi S. Anatomical Variations in the Branching Pattern of Human Aortic Arch: A Cadaveric Study from Central India. Int Sch Res Notices 2013; 828969.

3. Gaikwad MR, ,Patil KS, Tirpude AP , Wakode NS. Anomalous High Transverse Course of Brachiocephalic Trunk and its Clinical Significance: A Rare Case Report. J Clin Diagn Res 2018; 12(3):AD03-AD04 DOI: $10.7860 / J C D R / 2018 / 34387.11278$.

4. Iterezote AM, Medeiros AD, Barbosa Filho RC, Petrella S, Andrade Junior LC, Marques SR, Prates JC. Anatomical variation of the brachiocephalic trunk and common carotid artery in neck dissection. Int J Morphol 2009; 27(2):601-03.
5. Sharma NA, Garud RS. Dangerous course of the brachiocephalic trunk and variations of arteries in the cervical region - a cadaveric case report. Eur J Anat 2012; 16 (1): 74-78.

6. Karunakaran K, Pandian DM, Ganesan R, Gobinath M. Anomalous Course of Right Common Carotid Artery in a Case of Papillary Carcinoma Thyroid. Indian J Surg 2013; 75(2):145-146.

7. Teli C, Kate NN, Paarthipan N. High division and variation in brachial artery branching pattern. J Dent Med Sci 2013; 3(6): 68-70

8. Jadhav SD, Pawar SE. High Division of Brachial Artery and Its Clinical Insight: A Case Report. Int J of Current Med and Applied Sci 2018; 19(1) : 27-29

9. Calisto J, Lissarrague M, Mansilla A, Russo AM, Ugon GA. Axillary origin of both radial and ulnar arteries : A cadaveric case report. Int J Anat Var 2018; 11(4):131-133 